\title{
PHYSICAL ACTIVITY, APPROACH-AVOIDANCE TEMPERAMENT AND DEPRESSIVE SYMPTOMS
}

\author{
Jose A. Cecchini, Javier Fernandez-Río, and Antonio Mendez-Gimenez \\ Educational Sciences Department, University of Oviedo, Spain
}

Original scientific paper

https://doi.org/10.26582/k.51.1.13

\begin{abstract}
:
The goal was to assess the connections between vigorous physical activity (VPA), approach-avoidance temperament and depressive symptoms. Two studies were conducted. Study 1, correlational, to assess the mediating role of both dimensions of temperament, the approach-avoidance contrast, between physical activity and depressive symptoms. Participants, 335 college students, completed the International Physical Activity Questionnaire, the Depressive Symptoms Scale (DSS) and the Approach-Avoidance Temperament Questionnaire (Ap-AvTQ). Results showed that the approach-avoidance contrast could be considered a potential mediator between VPA and depressive symptoms. The global model was significant, $\mathrm{F}(2,351)=3.22$, $\mathrm{p}<.001, \mathrm{R}^{2}=14.91 \%, \mathrm{R}^{2}$ adjusted $=14.42 \%$, and the bootstrapped upper and lower limits did not contain zero with the lower limit at -.05 and the upper at -14 , suggesting a connection between VPA and depressive symptoms mediated by the approach-avoidance contrast temperament. Study 2, longitudinal, to test if a physical activity program could produce changes in the approach-avoidance contrast temperament, manipulating the depressive symptoms. A VPA program was conducted with 149 college students. Participants completed the DSS and the Ap-AvTQ. The true intraindividual change modeling technique, a more direct approach to modeling interindividual differences in intraindividual change without using a control group, showed the participants' depressive symptoms were predicted through the mediation of the approach-avoidance contrast temperament $(\gamma=-.36, p<.001)$. VPA was positively linked to the approach-avoidance contrast temperament that was negatively connected to depressive symptoms, and negatively linked to the approach-avoidance contrast temperament that was positively connected to depressive symptoms. It seems possible to influence depressive symptoms through the approach-avoidance contrast temperament using VPA.
\end{abstract}

Key words: moderate-to-vigorous physical activity, MET, prevention

\section{Introduction}

The five-factor model of personality includes, apart from Extraversion, Conscientiousness, Openness, and Agreebleness, Neuroticism, which has been defined as the tendency of some individuals to experience negative emotions and stress (McRae $\&$ Costa, 2008). In a recent meta-analysis, "higher Neuroticism and lower Conscientiousness were further associated with more time spent in actual sedentary behaviors" (Sutin, et al., 2016, p. 24). Moreover, individuals high on Neuroticism tend to hold avoidance-related goals regarding physical activity practice, since they try to avoid looking bad to other individuals (Lochbaum, Litchfield, Podlog, \& Lutz, 2013). According to the World Health Organization (2012), depression, linked to Neuroticism, is a mental disorder that affects more than 350 million people worldwide. This term has been used refering to a continuum of affective distur- bances that include dysphoric mood, a syndrome or cluster of symptoms of psychological distress, and psychiatric disorders (Galambos, Leadbeater, $\&$ Barker, 2004). Depressive symptoms have been linked to negative effects such as increased risk of major depression (Horwarth, Johnson, Klerman, \& Weissman, 1992), functional impairments (Lynnes, et al., 2007) and social disfunction (Judd \& Paulus, 1996). However, they are often overlooked and attributed to daily life strains (Beardslee \& Knitzer, 2004). Negative consequences of depressive symptoms on individuals have led many researchers to investigate their development, the factors that influence them, and ways to prevent or mitigate their effects. Different preventive programs have been conducted to moderate this important public health problem, and some have showed success in the reduction of depressive symptoms, particularly those who used physical activity (Adamson, Ensari, 
\& Motl, 2015; Cecchini-Estrada, Mendez-Gimenez, Cecchini, Moulton, \& Rodriguez, 2015; Vallance, et al., 2011). However, physical activity intensity seems to be an important element to consider. Depressive symptom reductions were significantly larger among patients who met moderate or vigorous physical activity recommendations (Herring, Puetz, O'Connor, \& Dishman, 2012). Recent meta-analyses have found that vigorous physical activity was associated with lower odds of having higher depressive symptoms (Dogra, et al., 2017). Therefore, physical activity programs' intensity level could be considered a significant factor of achieving positive effects on individuals' depressive symptoms reduction.

Among other biological, psychological and/ or conductual traits, individuals who suffer from depression have low levels of neurotransmitters (Rethorst, Landers, Naoshi, \& Ross, 2011). Physical activity has been linked to an increase in these substances (Craft \& Perna, 2004). It has been observed as a moderator between them and depressive symptoms too (Cecchini-Estrada, Fernández Río, Méndez-Giménez, Carriedo, \& Arruza, 2017; Rethorst, et al., 2011). Melancon, Lorraine and Dionne (2012) support the idea that an increase in individuals' neurotransmitter levels could explain the antidepressant effects of physical activity. Similarly, changes in indiviuals' temperament have been linked to changes in their neurotransmitters (Posner, Rothbart, \& Sheese, 2007). Moreover, Gonda et al. (2009) found a connection between them and temperament, which led to depression. Therefore, a physical activity program capable of increasing these substances could have a positive effect on prosocial temperament (Craft \& Perna, 2004). There seems to be a connection between physical activity, depressive symptoms and temperament (Brammer \& Lee, 2013; Li \& Lee, 2014; Ono, et al., 2002). This connection, if confirmed, could have important therapeutic consequences for the treatment of depressive symptoms.

Temperament has been found to have a strong heritable component (Service et al., 2012), but it also has biological base (Bates, 1989). Its characteristics arise during infancy, and they are also influenced by maturation and experience (Henderson \& Wachs, 2007). Besides genetic and biological influence, the effect of contextual factors on individuals' temperament is also being studied by researchers (Wachs \& Kohnstamm, 2001). Moreover, temperament is presently viewed as a set of characteristics that have the potential to change over time in response to the context (Henderson \& Wachs, 2007; Wachs, 2006). However, a modest stability of temperament is expected across different contexts and over time (Henderson \& Wachs, 2007). Ono et al. (2002) conducted a genetic analysis of the dimensions of temperament and depressive symptoms and found that depression affected more people with high levels of fear of pain and reward dependant. These authors hypothesized that what could be really inherited in depression was the temperament that predisposed and derived into vulnerability to depression, manifestation of which was determined, ultimately, by environmental factors. They highlighted the importance of studying the dimensions of temperament more than those of depression. More than a decade ago, Elliot and Thrash (2002) acknowledged two dimensions of temperament (approach and avoidance), which were also identified as two dimensions of personality. Approach temperament has been defined as a general neurobiological sensitivity to positive stimuli, while avoidance temperament is considered a general neurobiological sensitivity to negative stimuli. Both include perceptual vigilance, affective reactivity and behavioural predisposition. Disfunction in any of these two dimensions has been considered a key element to understand depression (Spielberg, Heller, Silton, Stewart, \& Miller, 2011). More precisely, Davidson (1998) believed that it is associated with a deficit in the approach dimension. Depression has also been linked to a bias in the avoidance motivational system, produced by low activity levels of the approach system and high levels of the avoidance system, or both (Henriques $\&$ Davidson, 2000). Spielberg et al. (2011) found that depression correlated positively with avoidance temperament and negatively with approach temperament. Low possitive or negative emotionality has been seen as a strong affective temperamental risk leading to depression (Elovainio et al., 2015; Hankin, 2015). Li and Lee (2014) found that negative emotionality was linked to infant depression and neurobilological factors affecting neurotransmission. Moreover, Brammer and Lee (2013) discovered that neurotransmitters were tied to several dimensions of temperament: positively with negative emotionality and negatively with prosocial temperament. The mediating effect of temperament on depression seems to depend on its two dimensions (Henderson \& Wachs, 2007). The next step would be to find if these dimensions can be modified by contextual factors, such as physical activity, to induce depression reduction.

Based on the several ideas mentioned above: (1) temperament is what predicts, predisposes and derives into vulnerability to depression (Ono, et al., 2002); (2) a link between cerebral neurotransmitters and affective temperament that lead to depression has been found (Gonda, et al., 2009); (3) several dimensions of temperament can mediate between cerebral neurotransmitters and depression (Li \& Lee, 2014); (4) temperament is considered a group of individual predispositions with the potential to change over time as a response to changes in the context such as physical activity (Wachs, 2006); (5) avoidance and approach temperament have 
been positively and negatively linked to depression, respectively (Spielberg, et al., 2011); and (6) physical activity produces an increase in cerebral neurotransmitters (Craft \& Perna, 2004), which could positively affect temperament and depressive symptoms, the following hypotheses were established: Hypothesis 1 - approach temperament will be related to lower levels of depressive symptoms, avoidance temperament will be linked to higher levels of depressive symptoms, and VPA will be connected to lower levels of depressive symptoms; Hypothesis 2 - approach temperament will be positively linked to VPA, while avoidance temperament will be negatively linked to VPA; Hypothesis 3 - VPA will moderate the temperament-depression relationship. To test these hypotheses, two studies were designed: study one, transversal, was conducted to assess the mediating role of both temperament dimensions between physical activity and depressive symptoms. To test if a physical activity program could produce changes in the dimensions of temperament manipulating the depressive symptoms, the second study, longitudinal, was conducted.

\section{Study 1}

\section{Participants}

Three hundred fifty-three $(\mathrm{N}=353)$ university students, aged between 19 and 29 years $(M=20.12$, $\mathrm{SD}=2.35$ ) agreed to participate; 176 were males and 177 females. Ten participants missed $>8 \%$ data. Therefore, they were imputed using values derived from a multiple regression, where three item scores from the same congeneric set of indicators were used as the predictor variables (Byrne, 2005). Participants' mean depressive symptomatology score was 24.28 , which could be considered moderate. Choi, Patten, Gillin, Kaplan, and Pierce (1997, p. 43) defined "adolescents who achieve a score of 29 or greater as having notable depressive symptoms". Using the same cutoff score, $11.90 \%$ of the sample could be considered as having notable depressive symptoms. A written informed consent was obtained from all participants as well as the permission from the Ethics committee of the researchers' university.

\section{Instruments}

International Physical Activity Questionnaire (IPAQ). The short version of the IPAQ, the "7-day recall" (Craig, et al., 2003) was used to assess vigorous physical activity (VPA). Several studies agree that VPA is a better predictor of a decrease in depressive symptoms than a lighter physical activity (Lee \& Russell, 2003; Wise, Adams-Campbell, Palmer, \& Rosenberg, 2006). The short version of the IPAQ has been validated in individuals aged 18-65 years and is considered a reliable instrument
(Craig, et al., 2003). VPA was assessed using the metabolic equivalent of task (MET) in accordance with Ainsworth et al. (2000), and it was expressed in MET-minutes/week. METs are multiples of the basal metabolic rate, and they measure energy needs. In this study, we used METs-VPA.

Depressive symptoms. A six-item self-report scale from Kandel and Davies (1982) was used (Choi, et al., 1997). It measures six depressive symptoms over the last twelve months. Questions referred to social activities that could affect individuals' health (i.e., Felt unhappy, sad or depressed?). Following Hambleton, Merenda and Spielberger (2005), all items were translated into Spanish by a specialist, and then again into English to test their conformity with the original ones. Two experts assessed all the items and approved their adequacy to measure the approach and avoidance achievement goals. Participants answered on a 4-point Likert-like scale: "never", "rarely", "sometimes" and "often". Self-reported depressive symptoms are the strongest predictive measure for the appearance of major depressive disorder in youth (Kovacs \& Lopez-Duran, 2010). To provide a depressive symptomatology score, the responses to the items were added. Finally, these scores were increrased by 10 points to produce a range between 16 and 34 points (Choi, et al., 1997). These scores were used to provide information on the participants' depressive symptomatology. Mean and standard deviation of the original scores were used for statistical analyses. The Cronbach's alpha coefficient was acceptable in this study $(\alpha=.84)$.

Approach-Avoidance Temperament Questionnaire (ATQ; Elliot \& Thrash, 2010). It is divided in two dimensions: six items assess approach temperament (i.e., "It doesn't take a lot to get me excited and motivated"), and six items assess avoidance temperament (i.e., "It is easy for me to imagine bad things that might happen to me"). Cronbach's alpha coefficients in the present study were .84 and .83 , respectively. Participants responded on a 7-point Likert scale from 1: "Totally disagree" to 7: "Totally agree". Approach and avoidance temperament indexes were calculated. Again, we followed Hambleton et al.'s (2005) procedure to ensure the translated version's adequacy to measure approach and avoidance temperament.

\section{Data analysis}

Exploratory and descriptive analysis and bivariate correlations were calculated to assess the connections included in hypotheses 1 and 2. Using SPSS macro INDIRECT (Preacher \& Hayes, 2008), the initial hypothesis, i.e., the mediator role of both dimensions of temperament in METs-VPA and depressive symptoms, was assessed. This method was used because it provides an overview of simple and multiple mediation, and it helps explore different 
approaches that can be used to investigate indirect processes, as well as the methods to contrast two or more mediators within a single model. Therefore, the direct and global effects of METs-VPA on depressive symptoms, the indirect effects through the approach and avoidance temperament variables, and the specific direct effects of this variable on depressive symptoms were assessed. Since data distribution was not normal, bootstrapping methods were used, with a resample of 5000 bootstraps. Follow-up analyses were performed based on gender and age (Mavroveli, Petrides, Rieffe, \& Bakker, 2007). Following previous research (Stoeber \& Crombie, 2010), both temperaments were first standardized prior to the contrast calculation into a single variable: approach-avoidance contrast $=$ zapproach - zavoidance temperament. Although not with temperament, Lochbaum and Gottardy's (2015) meta-analysis showed that the variable App-Avoid could be the best predictor of sport performance. Furthermore, Lochbaum (2014) examined whether athlete rated goal value and efficacy to achieve pre-contest goal mediated the relationship between approach-avoidance temperament and pre-contest achievement goals. Bootstrapped ninety-five percent confidence intervals $(95 \% \mathrm{CI})$ were used to evaluate significance of the indirect effects for each hypothesized path.

\section{Results}

\section{Preliminary analysis}

Table 1 shows that participants scored higher in the approach than in the avoidance temperament. Correlations were observed among all variables. METs-VPA was positively related to the approach temperament and negatively to the avoidance temperament and depressive symptoms, which were positively linked to the avoidance temperament and negatively to the approach temperament.

\section{Mediation analysis}

To test hypothesis 3, SPSS macro INDIRECT was used. Figure 1 shows that the global model was significant $F(2,351)=3.22, p<.001, R 2=14.91 \%$, $\mathrm{R} 2$ adjusted $=14.42 \%$ and the bootstrapped upper and lower limits did not contain zero, with the lower limit at -.05 and the upper at -.14 , suggesting a connection between VPA and depressive symptoms mediated by the approach-avoidance contrast temperament. The $\mathrm{c}$ in Figure 1 represents the total effect of the approach-avoidance contrast on depressive symptoms, while the c' shows the direct effect of the approach-avoidance contrast on the same variable.

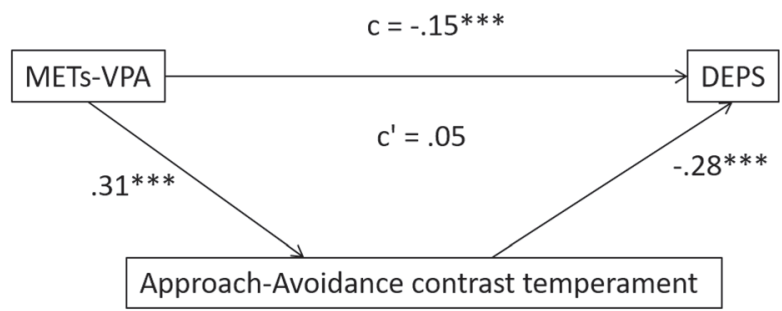

Figure 1. METs-VPA, approach-avoidance contrast temperament and depressive symptoms $\left({ }^{* * *} p<.001\right)$.

\section{Study 2}

\section{Participants}

One hundred forty-six $(\mathrm{n}=146)$ university students, aged between 18 and 31 years, agreed to participate. They were chosen through a rigorous selection process (Figure 2). Four missed $>8 \%$ data. Therefore, they were imputed with values derived from a multiple regression where three item scores from the same congeneric set of indicators were used as the predictor variables (Byrne, 2005). Participants' mean depressive symptomatology score was 24.76 , which could be considered moderate (Choi, et al., 1997). Again, using the same cutoff score of $29,19.38 \%$ of the sample could be considered as having notable depressive symptoms. A written informed consent was obtained from all participants, as well as the permission from the Ethics Comittee of their University.

\section{Procedure}

All participants experienced a supervised VPA program: 45 minutes, 3 days a week, over a time span of 12 weeks. VPA programs (aprox. $>6$ MET) require an intense exertion and produce a significant heart rate increase. A physical education teacher with more than 10 years of teaching experience supervised the VPA program. It was designed based on these strategies: a) global, open

Table 1. Descriptive statistics and bivariate correlations among variables

\begin{tabular}{lcccccc}
\hline & $\mathrm{M}$ & $\mathrm{DS}$ & 1 & 2 & 3 & 4 \\
\hline 1. AvT & 3.36 & 1.33 & 1 & & & \\
2. AppT & 5.61 & .84 & $.13^{*}$ & 1 & & \\
3. METs-VPA & 1779.55 & 2019.83 & -.15 & $.17^{* *}$ & 1 & $-.15^{* *}$ \\
4. DEPS & 2.38 & .67 & $.33^{* *}$ & $-.17^{* *}$ & 1 \\
\hline
\end{tabular}

Note. METs-VPA - MET minutes/week-vigorous physical activity; AvT - avoidance temperament; AppT - approach temperament; DEPS - depressive symptoms; $M$ - mean; SD - standard deviation. " $p<.05 ;$; $p<.01$. 


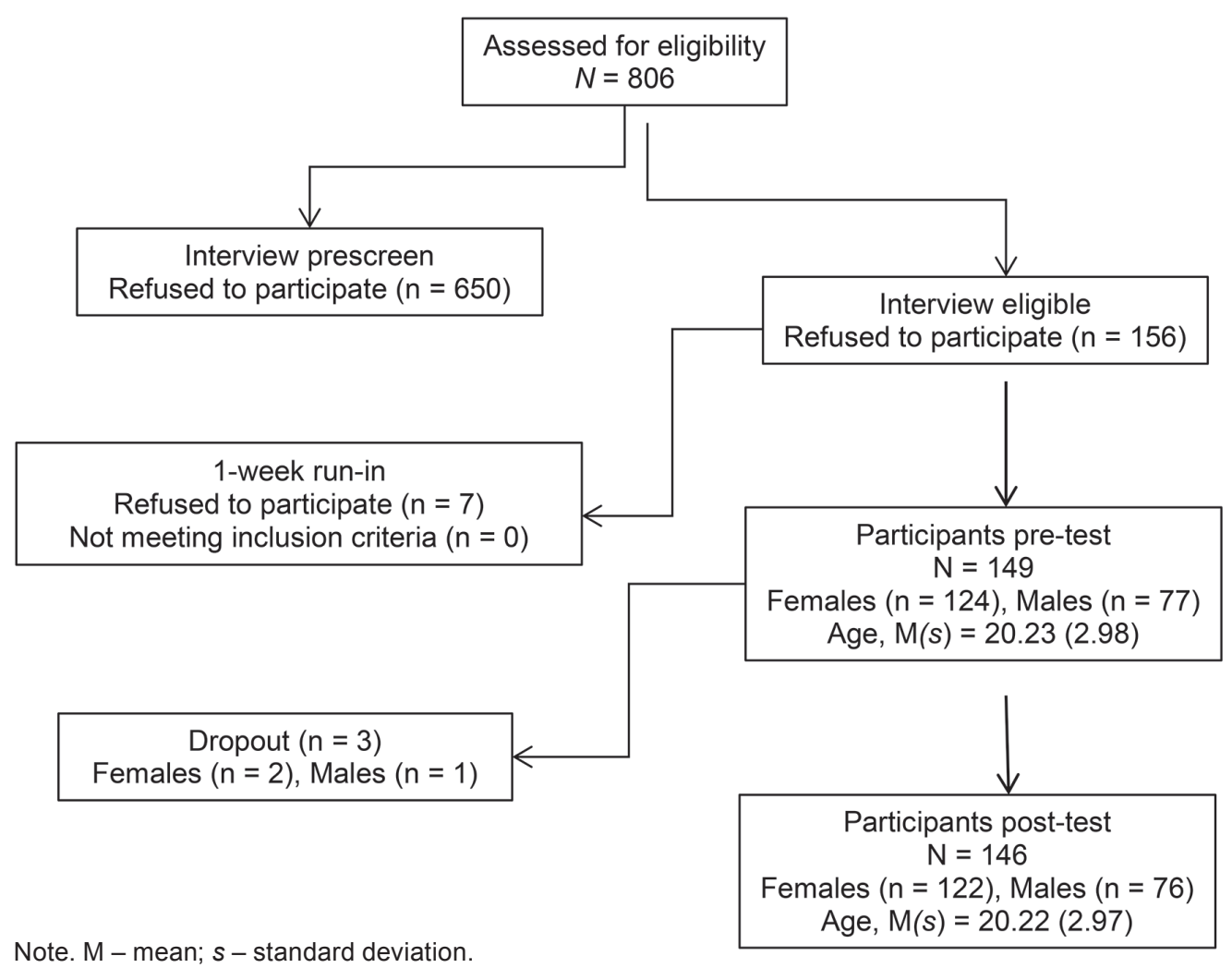

Figure 2. Flow of participants' selection.

tasks; b) varied activities, which were whenever there was a decrease in the participants' work intensity level; c) motivating tasks adapted to fit each student's needs and interests; d) promotion of group work and reciprocal help; and e) keeping each task's intensity level vigorous (heavy breathing, elevated pulse, sweat...). Prior to (T1) and at the end of the VPA program (T2), all participants answered a questionnaire to assess depressive symptoms and temperament. They were asked to be totaly honest, since complete anonymity and confidentiality were guaranteed.

\section{Instruments}

Depressive symptoms. A six-item scale by Kandel and Davies (1982) was used (Choi, et al., 1997). It has been described in study 1 . Cronbach's alpha coefficients were .80 at $\mathrm{T} 1$ and .83 at T2.

Approach-Avoidance Temperament Questionnaire (ATQ; Elliot \& Thrash, 2010). It has been described in study 1 . Cronbach's alpha coefficients were .81 at $\mathrm{T} 1$ and .83 at T2 for the ApproachTemperament, and .79 at $\mathrm{T} 1$ and .81 at $\mathrm{T} 2$ for the Avoidance-Temperament.

\section{True intraindividual change model}

The True Intraindividual Change model (TIC), within the Structural Equation Model (SEM), was used to assess changes in the approach-avoidance contrast temperament and depressive symptoms from pre- to post-tests in each individual partic- ipant (Steyer, 2005; Steyer, Partchev, \& Shannahan, 2000). The true intraindividual change (TIC) model represents a more direct approach to modelling interindividual differences in intraindividual change without using a control group (Steyer, Eid, \& Schwenkmezger, 1997). The TIC is based on two basic ideas: a) at least two observed variables measure the same latent variable at two times, and b) the assessment model (the regression coefficients of the observed values in the latent variables) is invariant across time. A parceling approach procedure was used to form two indicators per construct (Little, Cunningham, Shahar, \& Widaman, 2002), as well as an internal consistency approach to create parcels for the variables analyzed such that measurement items of the same subscale were combined in the same parcel.

To test the hypothesis that the model was invariant across time, a confirmatory factor analysis was conducted using the program EQS 6.2. (Bentler, 2006). Since preliminary data showed a substantial multivariate kurtosis, analyses were based on the Satorra-Bentler scaled chi-square statistics (S-B $\chi^{2}$; Satorra \& Bentler, 1988). The sample's goodness-of-fit was conducted using multiple criteria (Byrne, 2008): the Comparative Fit Index (*CFI; Bentler, 1990), and the Root Mean-Square Error of Approximation (*RMSEA; Browne \& Cudeck, 1993). Results supported the factorial invariance. Comparing the restricted model (S-B $\chi^{2}[14]=17.05$, ${ }^{*} \mathrm{CFI}=.996, \mathrm{SRMR}=.049, *$ RMSEA $=.033 \quad[90 \%$ 
$\mathrm{IC}=.000, .080])$ and the model with no invariance restrictions $\left(\mathrm{S}-\mathrm{B} \chi^{2}[12]=16.61,{ }^{*} \mathrm{CFI}=.993\right.$, SRMR $=.036$, RMSEA $=.044[\mathrm{IC}=.000, .0791])$, the fit to the model did not improve significantly $\left(\triangle \mathrm{S}-\mathrm{B} \chi^{2}\right.$ (3) $=.46$, n.s.).

The second stage required testing a baseline model. In this model (Figure 3), it was hypothesized that the observed variables in the T2 could predict their respective latent factors in the $\mathrm{T} 1$ and the T2. The scores of each latent variable in T2 had been regressed to the corresponding latent variables at $\mathrm{T} 1$. This configuration transforms the latent factor at T2 in scores of the true intraindividual change across time, which allows testing the proposed model. Synchronic correlations among latent variables for each group of exogenous and endogenous factors were performed (they are not shown in Figure 3). Error variables were also correlated (they are shown in Figure 3), since they did not invalid the model (Steyer, 2005). Factorial loadings were considered invariant across time.

\section{Results}

\section{Preliminay analysis}

Table 2 shows that significant changes were observed between T1 and T2: the approach-avoidance contrast temperament's scores increased, while the depressive symptoms' scores decreased. Table 3 also shows correlations among latent variables. Pattern changes between depressive symptoms and temperament are connected.

\section{Testing the proposed model}

Results from the SEM analysis showed that the model provided a good fit $\left(\mathrm{S}-\mathrm{B} \chi^{2}[14]=18.68\right.$, $\mathrm{p}=.165,{ }^{*} \mathrm{CFI}=.993, \mathrm{SRMR}=.045, * \mathrm{RMSEA}=.041$ $[90 \%(\mathrm{IC})=.000, .085])$. The true intraindividual changes in the depressive symptoms were predicted by intraindividual changes in the approach temperament $(\gamma=-.36, p=.001)$.

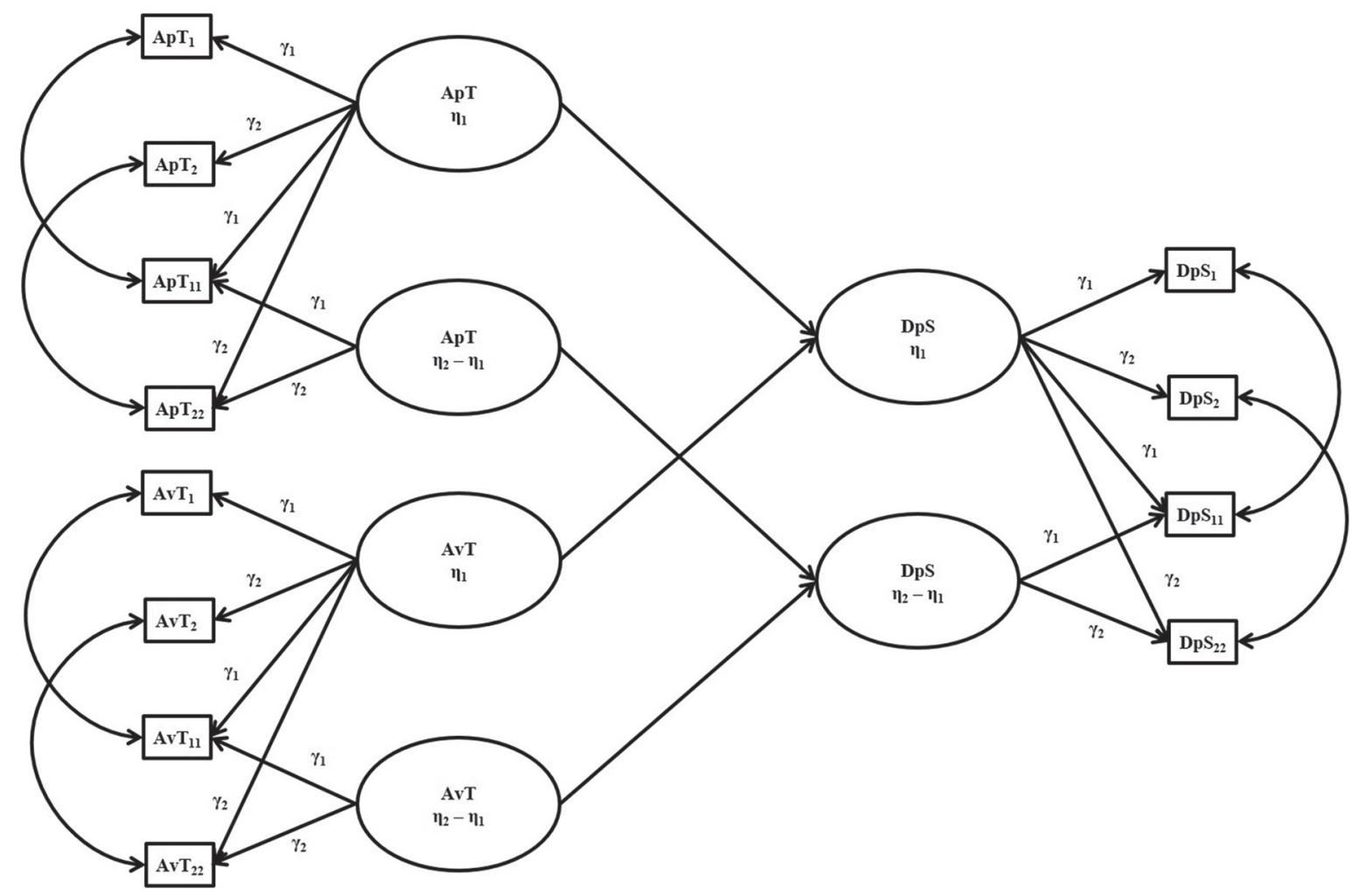

Figure 3. Theoretical model.

Table 2. Descriptive statistics and correlations between the latent and the modified latent variables

\begin{tabular}{|c|c|c|c|c|c|c|}
\hline & LM & SD & 1 & 2 & 3 & 4 \\
\hline 1. Approach-avoidance contrast $\eta_{1}$ & 1.98 & 1.35 & - & & & \\
\hline 2. $S D p \eta_{1}$ & 2.41 & .70 &,$- 33^{* *}$ & - & & \\
\hline 3. $\Delta$ approach-avoidance contrast $\left(\eta_{2}-\eta_{1}\right)$ & $.54^{* * *}$ & .85 & $-.15^{* *}$ & $.22^{* *}$ & - & \\
\hline 4. $\triangle$ DEPS $\left(\eta_{2}-\eta_{1}\right)$ & $.23^{* * * *}$ & .68 & -.06 & $-.40^{* *}$ & $-.41^{* *}$ & - \\
\hline
\end{tabular}

Note. LM - latent mean; SD - standard deviation; $\Delta$ - intraindividual change $\left(\eta^{2}-\eta^{1}\right)$; DEPS - depressive symptoms. "p<.05; "* $p<.01$. 


\section{Discussion and conclusion}

The goal of the present study was to assess the connections between vigorous physical activity (VPA), approach-avoidance temperament and depressive symptoms. The following hypotheses were set: (1) the approach temperament will be related to lower levels of depressive symptoms, the avoidance temperament will be linked to higher levels of depressive symptoms, and VPA will be connected to lower levels of depressive symptoms; (2) the approach temperament will be positively linked to VPA, while the avoidance temperament will be negatively linked to VPA; and (3): VPA will moderate the temperament-depression relationship. Results of the study confirmed the hypotheses since VPA was positively linked to the approach temperament, which was negatively linked to depressive symptoms. Vigorous physical activity was also negatively linked to the avoidance temperament, which was positively linked to depressive symptoms. These results could have important theoretical and practical implications. On the one hand, they reinforce the idea that physical activity could be very useful in depressive symptoms' reduction (Vallance, et al., 2011). They also help understand how physical activity can mediate through both dimensions of temperament (approach and avoidance) to make an impact on depressive symptoms. A possible explanation could be that physical activity can have physiological benefitial influence on them due to an increase in cerebral neurotransmitters (Craft \& Perna, 2004). Major depressive disorders have been connected to physicological changes in brain structure and function related to chronic neuroinflamation (Uher, Payne, Pavlova, $\&$ Perlis, 2014). Recent reviews have pointed out numerous inflammation-altering effects derived from physical activity or exercise practice: “... elevating the expression of neurotrophic factors, enhancing the release of neurotransmitters and increasing neurogenesis. Attenuation of glial cell activation and the resulting reduction of inflammation is an additional benefit of PA [physical activity] and exercise" (Spielman, Little, \& Klegeris, 2016, p. 26). In study 1 , the connection between VPA and depressive symptoms seemed to be mediated by both dimensions of temperament, which has been observed in previous studies (Brammer \& Lee, 2013; Gonda, et al., 2009; Li \& Lee, 2014; Ono, et al., 2002). A possible explanation for this mediation is that temperament is what truly predisposes and derives in vulnerability to depression. Therefore, if the individuals' temperament is modified, their depressive symptoms could also be modified. Li and Lee (2014) observed that temperament can mediate between neurotransmitters and depression, and, consequently, between physical activity, which increases cerebral neurotransmitters, and depressive symptoms.
On the other hand, PA intensity seems to be an important element to consider when implementing physical activity or exercise programs for individuals who suffer from depression. Depressive symptoms' reductions were significantly larger among patients who met moderate or vigorous physical activity recommendations (Herring, et al., 2012). Recent meta-analyses have linked vigorous physical activity to lower odds of having higher depressive symptoms (Dogra, et al., 2017). Moreover, high scores of neuroticism have been inversely associated with moderate-to-vigorous physical activity (Rhodes \& Smith, 2006)

Findings from the present study could have significant consequences. They have showed a new approach to fight depressive symptoms through the therapeutic interaction of both dimensions of temperament. However, it is necessary to solve one dilemma: is it possible to modify those dimensions considering that they are relatively stable traits of an individual's personality? Nowadays, the genetic influence on temperament is being questioned by the evidences of contextual effects on its dimensions (Henderson \& Wachs, 2007; Wachs \& Kohnstamm, 2001). Individual predispositions could change due to the adaptation process of the individual to a socio-cultural context (Wachs, 2006). To test this hypothesis, study 2 was conducted. The goal was to assess if a VPA program could modify the approach-avoidance contrast temperament, which sequentially could have an effect on the individual's depressive symptoms. Results of the longitudinal study supported the initial hypothesis. In a relatively short period of time, the approachavoidance contrast temperament scores significantly increased. Previous research has indicated that a modest stability of temperament is expected across different contexts and over time (Henderson $\&$ Wachs, 2007). To our knowledge, there are no published studies to which the findings of the present study can be compared. Temperament has always been considered a fairly stable personality trait, which was not supported in the present study, since significant changes were obtained. This indicates that, at least temporary, alterations in the individuals' temperament were achieved. The VPA program could have produced this effect. This is highly speculative at this time, and further research is needed to clarify this issue. Moreover, findings from the present study indicate that not only the intensity levels should be considered in PA programs. The motivational processes originated in those programs should also be assessed. Future studies should consider the effects of individual versus group physical activity programs on temperament and depression, and the incidence of motivational frameworks such as the Self-Determination Theory or motivational strategies such as TARGET (Epstein, 1988). 
The present study holds some limitations. First, the sample size in study 2 could be considered limited. Similar studies should be conducted with large samples. Second, the VPA program's intensity level was not measured. Future research works should use accelerometers or heart monitors to assess intensity level of the physical activity program used. Subsequent research works on biological/physiological indicators of temperament to suplement the present findings should also be conducted.
In conclusion, it seems possible to influence depressive symptoms through temperament. A true intraindividual change on depressive symptoms was observed through the changes in the approachavoidance temperament after experiencing a VPA program. Physical activity could influence an individual's temperament by decreasing his/her fear, anxiety or worry, and increasing his/her energy, enthusiasm, and motivation through positive experiences.

\section{References}

Adamson, B.C., Ensari, I., \& Motl, R.W. (2015). The effect of exercise on depressive symptoms in adults with neurological disorders: A systematic review and meta-analysis. Archives of Physical Medicine and Rehabilitation, 96(7), 1329-1338. doi:10.1016/j.apmr.2015.01.005

Ainsworth, B.E., Haskell, W.L., Whitt, M.C., Irwin, M.L., Swartz, A.M., Strath, S.J., ..., \& Leon, A.S. (2000). Compendium of physical activities: An update of activity codes and MET intensities. Medicine and Science in Sports and Exercise, 32(9), Suppl., 498-516.

Bates, J.E. (1989). Concepts and measures of temperament. In G.A. Kohnstamm, J.E. Bates \& M.K. Rothbart (Eds.), Temperament in childhood (pp. 3-26). Oxford, England: Wiley.

Beardslee, W.R., \& Knitzer, J. (2004). Mental health services: A family systems approach. In K. Maton, C. Shellenbach, B. Leadbeater \& A. Solarz (Eds.), Investing in children, youth, families, and communities: Strengths-based research and policy (pp. 147-183). Washington, DC: American Psychological Association.

Bentler, P.M. (2006). EQS 6 structural equations program manual. Encino, CA: Multivariate Software.

Brammer, W.A., \& Lee, S.S. (2013). Prosociality and negative emotionality mediate the association of serotonin transporter genotype with childhood ADHD and ODD. Journal of Clinical Child and Adolescent Psychology, 42, 809-819.

Browne, M.W., \& Cudeck, R. (1993). Alternative ways of assessing model fit. In K.A. Bollen and J.C. Long (Eds), Structural equation models (pp. 136-162). London: Sage.

Byrne, B.M. (2005). Testing for multigroup equivalence of a measuring instrument: A walk through the process. Psicothema, 20(4), 872-882.

Cecchini-Estrada, J.A., Fernández Río, J., Méndez-Giménez, A., Carriedo., A, \& Arruza, J.A. (2017). A selfdetermination approach to the understanding of the impact of physical activity on depressive symptoms. Stress and Health, 33(5), 600-607. doi: 10.1002/smi.2744

Cecchini-Estrada, J.A., Mendez-Gimenez, A., Cecchini, C., Moulton, M., \& Rodriguez, C. (2015). Exercise and Epstein's TARGET for treatment of depressive symptoms: A randomized study. International Journal of Clinical and Health Psychology, 15(3), 191-199. doi:10.1016/j.ijchp.2015.05.001

Choi, W.S., Patten, C.A., Gillin, J.C., Kaplan, R.M., \& Pierce, J.P. (1997). Cigarette smoking predicts development of depressive symptoms among US adolescents. Annals of Behavioral Medicine, 19, 42-50. doi: 10.1007/BF02883426

Craft, L.L., \& Perna, F.M. (2004). The benefits of exercise for the clinically depressed. Journal of Clinical Psychiatry, 6, 104-111.

Craig, C.L., Marshall, A.L., Sjöström, M., Bauman, A.E., Booth, M.L., Ainsworth, B. E., ..., \& Oja, P. (2003). The IPAQ Consensus Group and the IPAQ Reliability and Validity Study Group. International Physical Activity Questionnaire (IPAQ): 12-country reliability and validity. Medicine and Science in Sports and Exercise, 35, 1381-1395.

Davidson, R.J. (1998). Affective style and affective disorders: Perspectives from affective neuroscience. Cognition and Emotion, 12, 307-330.

Dogra, S., Macintosh, L., O’Neill, C., D’Silva, C., Shearer, H., Smith, K., \& Coté, P. (2017). The association of physical activity with depression and stress among post-secondary students: A systematic review. Mental Health and Physical Activity. doi: 10.1016/j.mhpa.2017.11.001

Elliot, A.J., \& Thrash, T. (2002). Approach-avoidance motivation in personality: Approach and avoidance temperaments and goals. Journal of Personality and Social Psychology, 82(5), 804-818.

Elliot, A.J., \& Thrash, T. (2010). Approach and avoidance temperament as basic dimensions of personality. Journal of Personality, 78, 865-906.

Elovainio, M., Jokela, M., Rosenström, T., Pulkki-Råbäck, L., Hakulinen, C., Josefsson, K., ...., \& Keltikangas-Järvinen, L. (2015). Temperament and depressive symptoms: What is the direction of the association? Journal of Affective Disorders, 170(1), 203-212. 
Epstein, J. (1988). Effective schools or effective students? Dealing with diversity. In R. Haskins \& B. MacRae (Eds.), Policies for America's public schools (pp. 89-126). Norwood, NJ: Ablex.

Galambos, N., Leadbeater, B., \& Barker, E. (2004). Gender differences in and risk factors for depression in adolescence: A 4-year longitudinal study. International Journal of Behavioral Development, 28(1), 16-25.

Gonda, X., Fountoulakis, K.N., Juhasz, G., Rihmer, Z., Lazary, J., Laszik, A., ..., \& Bagdy, G. (2009). Association of the s allele of the 5-HTTLPR with neuroticism-related traits and temperaments in a psychiatrically healthy population. European Archives of Psychiatry and Clinical Neuroscience, 259, 106-113.

Hambleton, R.K., Merenda, P.F., \& Spielberger, C. D. (2005). Adapting educational and psychological tests for crosscultural assessment. Mahwah, NJ: Lawrence Erlbaum.

Hankin, B.J. (2015). Depression from childhood through adolescence: Risk mechanisms across multiple systems and levels of analysis. Current Oppinion in Psychology, 4, 13-20.

Henderson, H.A., \& Wachs, T.D. (2007). Temperament theory and the study of cognition - Emotion interactions across development. Developmental Review, 27, 396-427.

Henriques, J.B., \& Davidson, R. (2000). Decreased responsiveness to reward in depression. Cognition and Emotion. 14, 711-724.

Herring, M.P., Puetz, T.W., O’Connor, P.J., \& Dishman, R.K. (2012). Effect of exercise training on depressive symptoms among patients with a chronic illness: A systematic review and meta-analysis of randomized controlled trials. Archives of Internal Medicine, 172(2), 101-111.

Horwath, E., Johnson, J., Klerman, G.L., \& Weissman, M.M. (1992). Depressive symptoms as relative and attributable risk factors for first-onset major depression. Archives of General Psychiatry, 49(10), 817-823.

Judd, L.L., \& Paulus, M.P. (1996). Socioeconomic burden of subsyndromal depressive symptoms and major depression in a sample of the general population. American Journal of Psychiatry, 153, 1411-1417.

Kandel, D.B., \& Davies, M. (1982). Epidemiology of depressive mood in adolescents: An empirical study. Archives of Geneneral Psychiatry, 39, 1205-1212.

Kovacs, M., \& Lopez-Duran, N. (2010). Prodromal symptoms and atypical affectivity as predictors of major depression in juveniles: Implications for prevention. Journal of Child Psychology and Psychiatry, 51(4), 472-496.

Lee, C., \& Russell, A. (2003). Effects of physical activity on emotional well-being among older Australian women: Cross-sectional and longitudinal analyses. Journal of Psychosomatic Research, 54(2), 155-160.

Li, J.J., \& Lee, S.S. (2014). Negative emotionality mediates the association of 5-HTTLPR genotype and depression in children with and without ADHD. Psychiatric Research, 215, 163-169.

Little, T.D., Cunningham, W.A., Shahar, G., \& Widaman, K.F. (2002). To parcel or not to parcel: Exploring the question, weighing the merits. Structural Equation Modeling, 9, 151-173. doi:10.1207/ S15328007SEM0902_1

Lyness, J.M., Kim, J., Tang, W., Tu, X., Conwell, Y., King, D.A., \& Caine, E.D. (2007). The clinical significance of subsyndromal depression in older primary care patients. American Journal of Geriatric Psychiatry, 15(3), 214-223.

Lochbaum, M. (2014). Approach-avoidance temperaments, goal value and efficacy, and pre-performance achievement goal contrasts: A test of mediation. Naučno-teoretičeskij Žurnal "Učenije Zapiski”" (Scientific Theory Journal), 117 (11), 213-218. doi: 10.5930/issn.1994-4683.2014.11.117

Lochbaum, M., \& Gottardy, J. (2015). A meta-analytic review of the approach-avoidance achievement goals and performance relationships in the sport psychology literature. Journal of Sport and Health Science, 17, 1-10. doi: 10.1016/j.jshs.2013.12.004

Lochbaum, M., Litchfield, K., Podlog, L., \& Lutz, R. (2013). Extraversion, emotional instability, and self-reported exercise: The mediating effects of approach-avoidance achievement goals. Journal of Sport and Health Science, 2, 176-183. doi: 10.1016/j.jshs.2012.08.002

Mavroveli, S., Petrides, K.V., Rieffe, C., \& Bakker, F. (2007). Trait emotional intelligence, psychological well-being and peer-rated social competence in adolescence. British Journal of Developmental Psychology, 25, 263-275.

McCrae, R.R., \& Costa, P.T. (2008). The Five-Factor Theory of personality. In O.P. John, R.W. Robins \& L.A. Pervin (Eds.), Handbook of personality: Theory and research ( $3^{\text {rd }}$ ed.). New York: Guilford Press.

Melancon, M.O., Lorrain, D., \& Dionne, I.J. (2012). Exercise increases tryptophan availability to the brain in older men aged 57-70 years. Medicine and Science in Sports and Exercise, 44(5), 881-887.

Ono, Y., Ando, J., Onoda, N., Yoshimura, K., Momose, T., Hirano, M., \& Kanba, S. (2002). Dimensions of temperament as vulnerability factors in depression. Mollecular Psychiatry, 7, 948-953.

Posner, M.I., Rothbart, M.K., \& Sheese, B.E. (2007). Attention genes. Developmental Science, 10, 24-29.

Preacher, K.J., \& Hayes, A.F. (2008). Asymptotic and resampling strategies for assessing and comparing indirect effects in multiple mediator models. Behavior Research Methods, 40, 879-891.

Rethorst, C.D., Landers, D.M., Nagoshi, C.T., \& Ross, J.T. (2011). The association of 5-HTTLPR genotype and depressive symptoms is moderated by physical activity. Journal of Psychiatric Research, 45, 185-189. doi:10.1016/j. jpsychires.2010.05.007

Rhodes, R.E., \& Smith, N.E. (2006). Personality correlates of physical activity: A review and meta-analysis. British Jounal of Sports Medicine, 40(12), 958-965. 
Satorra, A., \& Bentler, P.M. (1988). Scaling corrections for statistics in covariance structure analysis (UCLA Statistics Series 2). Los Angeles: University of California at Los Angeles, Department of Psychology.

Service, S.K., Verweij, K.J.H., Lahti, J., Congdon, E., Ekelund, J., Hintsanen, M., ..., \& Freimer, N.B. (2012). A genomewide meta-analysis of association studies of Cloninger's Temperament Scales. Translational Psychiatry, 2(5), e116.

Spielberg, J.M., Heller, W., Silton, R.L., Stewart, J.L., \& Miller, G.A. (2011). Approach and avoidance profiles distinguish dimensions of anxiety and depression. Cognitive Therapy and Research, 35(4), 359-371. doi:10.1007/s10608011-9364-0

Spielman, L.J., Little, J.P., \& Klegeris, A. (2016). Physical activity and exercise attenuate neuroinflamation in neurological diseases. Brain Research Bulletin, 125, 19-29.

Steyer, R. (2005). Analyzing individual and average causal effects. Methodology, 1, 39-54.

Steyer, R., Eid, M., \& Schwenkmezger, P. (1997). Modeling true intraindividual change: True change as a latent variable. Methods of Psychological Research Online, 2(1), 21-33.

Steyer, R., Partchev, I., \& Shanahan, M.J. (2000). Modelling true intraindividual change in structural equation models: The case of poverty and children's psychosocial adjustment. In T.D. Little, K.U. Schnabel \& J. Baumert (Eds.), Modelling longitudinal and multilevel data: Practical issues, applied approaches, and specific examples (pp. 109-126). Mahwah, NJ: Erlbaum.

Stoeber, J., \& Crombie, R. (2010). Achievement goals and championship performance: Predicting absolute performance and qualification success. Psychology of Sport and Exercise, 11(6), 513-521. doi: 10.1016/j.psychsport.2010.07.007

Sutin, A.R., Stephan, Y., Luchette, M., Artese, A., Oshio, A., \& Terracciano, A. (2016). The five-factor model of personality and physical inactivity: A meta-analysis of 16 samples. Journal of Research in Personality, 63, 22-28

Uher, R., Payne, J.L., Pavlova, B., \& Perlis, R.H. (2014). Major depressive disorder in DSM-5: Implications for clinical practice and research of changes from DSM-IV. Depression and Anxiety, 31(6), 459-471.

Vallance, J.K., Winkler, E.A., Gardiner, P.A., Healy, G.N., Lynch, B.M., \& Owen, N. (2011). Associations of objectivelyassessed physical activity and sedentary time with depression. Preventive Medicine, 53, 284-288. doi: 10.1016/j. ypmed.2011.07.013

Wachs, T.D. (2006). The nature, etiology and consequences of individual differences in temperament. In T. LeMonda \& L. Balter (Eds.), Child psychology: A handbook of contemporary issues (2nd ed.) (pp. 27-52). New York: Garland.

Wachs, T.D., \& Kohnstamm, G.A. (Eds.) (2001). Temperament in context. Mahwah, NJ: Lawrence Erlbaum.

Wise, L.A., Adams-Campbell, L.L., Palmer, J.R., \& Rosenberg, L. (2006). Leisure time physical activity in relation to depressive symptoms in the black women's health study. Annals of Behavioral Medicine, 32(1), 68-76.

World Health Organization (WHO). (2012). Depression. A global public health concern. Retreived from: http://www. who.int/mental_health/management/depression/who_paper_depression_wfmh_2012.pdf on January $2^{\text {nd }}, 2017$.

Submitted: May 25, 2017

Accepted: March 1, 2018

Published Online First: March 25, 2019

Correspondence to:

Javier Fernandez-Río, Ph.D.

Educational Sciences Department,

University of Oviedo, Oviedo, Spain

c) Aniceto Sela, s/n, despacho 219

3305-Oviedo-Asturias-Spain

Phone: +34-985102850

e-mail: javier.rio@uniovi.es 